\title{
A INFLUÊNCIA DO ESTILO DE VIDA E DO TRATAMENTO MULTIDISCIPLINAR NO CONTROLE DA SÍNDROME DOS OVÁRIOS POLICÍSTICOS: UMA REVISÃO BIBLIOGRÁFICA
}

\author{
Amanda Rocha Vasconcelos' ${ }^{1}$ Anselmo Messias Ribeiro da Silva Junior ${ }^{2}$, Isabela Borges Santos ${ }^{3}$, \\ Rosy Aline Lopes de Oliveira ${ }^{3}$, Mariane Costa Santos de Tavares ${ }^{3}$, Ana Virgínia Figueira Dubois \\ Mendes $^{3}$, João Pedro Gomes Saback ${ }^{3}$, Lorena Vieira Fernandez de Araújo ${ }^{3}$, Catarine Fortunato \\ Ferraz $^{4}$, Rafaela Martins Alves Lacerda ${ }^{5}$ and Brenda Badaró Reis ${ }^{6}$
}

${ }^{1}$ Discente do Curso de Graduação de Medicina. Faculdade Santo Agostinho - FASA, Vitória da Conquista-Bahia. ${ }^{2}$ Docente do Curso de Graduação de Medicina. Faculdade Santo Agostinho - FASA, Vitória da Conquista-Bahia. ${ }^{3}$ Discente do Curso de Graduação de Medicina. Faculdade Santo Agostinho - FASA, Vitória da Conquista-Bahia. ${ }^{4}$ Discente do Curso de Graduação de Medicina. Faculdade Federal de Alagoas, Maceió-Alagoas. ${ }^{5}$ Discente do Curso de Graduação de Medicina. Faculdade

Pitágoras de Medicina de Eunápolis-FPME, Eunápolis-Bahia. ${ }^{6}$ Discente do Curso de Graduação de Medicina. Centro Universitário UNiFG, Guanambi-Bahia

\begin{tabular}{|c|c|}
\hline ARTICLE INFO & ABSTRACT \\
\hline $\begin{array}{l}\text { Article History: } \\
\text { Received } 15^{\text {th }} \text { January, } 2021 \\
\text { Received in revised form } \\
26^{\text {th }} \text { January, } 2021 \\
\text { Accepted } 21^{\text {st }} \text { February, } 2021 \\
\text { Published online } 26^{\text {th }} \text { March, } 2021\end{array}$ & $\begin{array}{l}\text { A Síndrome dos Ovários Policísticos (SOP) é descrita pela associação do hiperandrogenismo e de } \\
\text { alterações da função ovariana (anovulação, oligo-ovulação e/ou morfologia ovariana policística), } \\
\text { com prevalência estimada em } 6 \text { a } 19 \% \text { das mulheres na menacme. Esta síndrome possui } \\
\text { importância clínica por influenciar diretamente na qualidade de vida e no aumento do risco de } \\
\text { diversas doenças, como infertilidade, câncer de endométrio e transtornos de humor. Dessa forma, } \\
\text { o obietivo central foi realizar um levantamento da literatura acerca de como a qualidade de vida e }\end{array}$ \\
\hline $\begin{array}{l}\text { Key Words: } \\
\text { Síndrome dos Ovários Policísticos, } \\
\text { Equipe Multiprofissional, } \\
\text { Estilo de vida, } \\
\text { Tratamento multidisciplinar, } \\
\text { Saúde da Mulher. }\end{array}$ & $\begin{array}{l}\text { a equipe multiprofissional influenciam no controle da SOP. Foi realizada uma revisão narrativa da } \\
\text { literatura científica, por meio das bases de dados científicas. Os descritores utilizados na busca } \\
\text { foram: "Síndrome do Ovário Policístico", "Equipe Multiprofissional" e "Hiperandrogenismo". A } \\
\text { interdependência dos fenômenos biológicos, mentais, sociais e ambientais nessas pacientes } \\
\text { justifica um tratamento estratégico que alia medidas não farmacológicas, como a prática regular } \\
\text { de atividade física, reeducação alimentar, acompanhamento psicológico, suplementação } \\
\text { nutricional e fármacos como os anticoncepcionais, sensibilizadores à insulina, antiandrogênicos e } \\
\text { indutores de ovulação. Assim, torna-se necessário um atendimento multiprofissional continuado, } \\
\text { com objetivo de modificação da composição corporal, dos parâmetros metabólicos, } \\
\text { cardiovasculares, hormonais e da função reprodutiva. }\end{array}$ \\
\hline
\end{tabular}

Copyright $($ C 2021, Amanda Rocha Vasconcelos et al. This is an open access article distributed under the Creative Commons Attribution License, which permits unrestricted use, distribution, and reproduction in any medium, provided the original work is properly cited.

Citation: Amanda Rocha Vasconcelos, Anselmo Messias Ribeiro da Silva Junior, Isabela Borges Santos et al. "A influência do estilo de vida e do tratamento multidisciplinar no controle da síndrome dos ovários policísticos: uma revisão bibliográfica", International Journal of Development Research, $11,(03), 45349-45357$.

\section{INTRODUCTION}

A síndrome dos ovários policísticos (SOP) é uma desordem endócrina heterogênea, que atinge cerca de 6-19\% das mulheres em idade reprodutiva, sendo considerada a endocrinopatia mais frequente da população feminina. A mesma é caracterizada pela associação do hiperandrogenismo (clínico e/ou laboratorial) com alterações da função ovariana (anovulação, oligo-ovulação e/ou morfologia ovariana policística) (AZEVEDO et al., 2008; COMISSÃO NACIONAL DE INCORPORAÇÃO DE TECNOLOGIAS NO SUS, 2019). Diversos elementos têm sido associados a etiopatogenia da SOP, dispondo de fatores metabólicos pré e pós-natais, componentes genéticos e distúrbios endócrinos hereditários envolvidos, como a resistência à insulina $(\mathrm{RI})$ e o diabetes mellitus tipo 2, e fatores ambientais (dieta e exercício 
físico) (ROSA-E-SILVA, 2018). No contexto da saúde da mulher, esta síndrome possui importância clínica por ser responsável pela diminuição da qualidade de vida de uma parcela considerável de mulheres, com manifestações psiquiátricas, riscos aumentados de neoplasias e eventos cardiovasculares (SILVEIRA, 2008). Múltiplos fatores causais têm sido sugeridos na fisiopatologia da SOP. Não está claro, quais anomalias aciona o ciclo vicioso de anovulação, excesso de andrógenos ou hiperinsulinemia observado na SOP. Entretanto, sabe-se que ocorre uma anormalidade intrínseca na pulsatilidade do hormônio liberador de gonadotrofina ( $\mathrm{GnRH}$ do inglês Gonadotropin-ReleasingHormone), levando ao aumento da amplitude e frequência dos pulsos do hormônio luteinizante $(\mathrm{LH})$. No que se trata da hiperinsulinemia, ela estimula o aumento da produção de andrógenos nas células da teca ovariana, e também inibe ainda mais a produção da SHBG (do inglês, sex hormone-bindingglobulin), que é produzida no fígado, e tem como principal função o transporte dos hormônios sexuais no sangue, ocasionando o aumento da fração livre da testosterona (VILAR et al., 2016).

Dessa forma, a paciente com SOP necessita de atendimento multidisciplinar continuado visando à alteração da composição corporal, dos parâmetros metabólicos, cardiovasculares, hormonais e da função reprodutiva. A interdependência dos fenômenos biológicos, psicológicos, sociais e ambientais nessas pacientes justifica um tratamento multiprofissional estratégico que alia fármacos, exercícios físicos regulares e reeducação alimentar (SILVA-DE-SÁ, 2018). Contrariando essa lógica, existe um número insipiente de estudos avaliando a conduta multiprofissional na abordagem em pacientes com SOP o que dificulta o tratamento individualizado e adaptado aos conhecimentos disponíveis de cada área. Somando-se a isso, o uso exclusivo de fármacos para tratamento dessa síndrome acarreta melhora dos sintomas, porém deixa lacunas no tratamento da etiologia e promove maiores efeitos adversos. Dessa forma, é vital utilizar dos recursos terapêuticos propostos pelos diversos grupos de profissionais de saúde e adequar o cuidado de forma integral. Ademais, a necessidade evidente da promoção e prevenção da saúde cardiovascular como foco nos distúrbios relacionados à obesidade $\mathrm{e}$ sedentarismo por meio de medidas não-farmacológicas tornam-se essenciais para a diminuição do risco de eventos mórbidos cardiovasculares que são de grande relevância pela alta mortalidade. É notório, portanto, a necessidade de produção de conteúdo científico que aborde essa temática. Nesse contexto, o presente trabalho visa responder à pergunta: Como a atuação da equipe multiprofissional e a qualidade de vida influencia no controle da SOP?. O objetivo geral escolhido foi: Descrever como a qualidade de vida e a equipe multiprofissional influenciam no controle da SOP. Além disso, essa revisão conta com três objetivos específicos, sendo eles: Descrever os tratamentos disponíveis para a SOP; Relacionar como a prática regular de atividade física e a reeducação alimentar ajudam no controle da SOP; Verificar a importância da equipe multiprofissional no controle da SOP.

\section{MATERIAIS E MÉTODOS}

Este estudo adotou como estratégia metodológica, a revisão bibliográfica - onde foi optado por utilizar a revisão narrativa. A revisão bibliográfica, ou revisão da literatura, é a análise crítica, meticulosa e ampla das publicações correntes em uma determinada área do conhecimento (TRENTINI; PAIM, 1999).
A pesquisa bibliográfica procura explicar e discutir um tema com base em referências teóricas publicadas em livros, revistas, periódicos e outros. Busca também, conhecer e analisar conteúdos científicos sobre determinado tema (MARTINS; PINTO, 2001). Foi realizada uma busca eletrônica na Biblioteca Virtual em Saúde (BVS) a qual resultou em documentos das bases de dados como MEDLINE (Medical Literature Analysis and Retrieval System Online), BIREME (Biblioteca Regional de Medicina), LILACS (Literatura Latino-americana e do Caribe em Ciências da Saúde) e SCIELO (Scientific Electronic Library Online). Além da utilização das orientações da Federação Brasileira das Associações de Ginecologia e Obstetrícia (FEBRASGO) e do Ministério da Saúde. Os termos utilizados na busca possuem a terminologia em saúde consultados nos descritores em ciências da saúde (DeCS) sendo eles: "Síndrome do Ovário Policístico","Equipe Multiprofissional"e "Hiperandrogenismo" Sendo que as publicações utilizadas foram escritas na língua inglesa e portuguesa. Na primeira parte da busca, foram usados e examinados os descritores de forma isolada, o que se detectou haver muitas publicações sobre o assunto em questão. Já na segunda parte, foi feita a combinação dos descritores, a fim de se aproximar das produções científicas encontradas, ou seja, daquelas que poderiam colaborar para a elucidação dos objetivos apontados. Após a identificação dos artigos, foi feita a leitura na integra para a construção do estudo, sendo excluídos artigos por ser duplicados nas bases de dados e por não responder o objetivo do estudo. Por ser uma revisão de literatura não será necessário submeter ao Comitê de Ética em Pesquisa (CEP), mas será mantido todos os aspectos éticos para evitar plágios e cópias.

\section{RESULTADOS}

Introdução e equipe multiprofissional: Nos dias de hoje, o tratamento da SOP não objetiva apenas à abordagem das repercussões reprodutivas, como infertilidade, anovulação e hirsutismo, mas também é conduzida com o intuito de promover e prevenir danos contra a saúde cardiovascular. Dessa forma, as medidas não-medicamentosas, tem ganhado espaço, especialmente no que tange à orientação nutricional e prática regular de exercício e/ou atividade física, se tornando medidas mandatórias, associada à interrupção do tabagismo, controle do estresse e consumo moderado de álcool. Sendo que, essas estratégias devem ser realizadas de forma prolongada para serem realmente efetivas (AZEVEDO et al., 2008). Nesse contexto, o tratamento da SOP é dividido em medidas farmacológicas e não-farmacológicas, ele envolve a regularização dos ciclos menstruais, o controle dos sintomas hiperandrogênicos e a proteção endometrial (GOODMAN et al., 2015). Dessa forma, a terapêutica medicamentosa baseiase principalmente no uso de contraceptivos combinados, drogas antiandrogênicas, drogas sensibilizadoras de insulina e indutores de ovulação (BENETTI-PINTO, 2018). Para um tratamento efetivo alguns critérios deverão ser analisados, tais como à idade da mulher, à fase da vida reprodutiva, peso, desejo de gestação e associação com irregularidades do metabolismo glicídico. Como também, a seleção do fármaco antiandrogênico é feito ainda pela combinação desses aspectos com critérios como magnitude do hirsutismo, custo, efetividade da droga e efeitos adversos (BENETTI-PINTO, 2018). Mesmo com a disponibilidade de tantos artifícios científicos, ainda não é possível ao médico ginecologista, de forma isolada, abordar com competência todas as etapas 
necessárias desta complexa terapia. Existe a necessidade do apoio conjunto com outros especialistas da área da saúde como endocrinologista, dermatologista, psicólogo, nutricionista, educador físico, dentre outros. Não há dúvidas que os fenômenos biopsicossociais e ambientais são correlacionados e a conduta deve objetivar um atendimento holístico e multiprofissional. Portanto, é interessante constatar quais os sintomas possuem maior impacto na paciente para serem priorizados nos cuidados (SILVA-DE-SÁ, 2018). Por isso, a SOP é um ótimo exemplo de tratamento multidisciplinar. Por ser um distúrbio multifatorial, que requer orientações focadas em várias metas, terá sua terapêutica mais eficaz com assistência de vários profissionais de saúde. Objetivos variados necessitam de condutas distintas, e a criação de uma equipe multiprofissional permitirá a abordagem ideal, levando ao êxito no controle da mesma e dos demais fatores de riscos (MALACHIAS et al., 2017). Para Peduzzi (2001), o trabalho em equipe é efetuado por meio da relação recíproca entre o processo técnico e a troca dos profissionais, configurando, por intermédio do diálogo, uma prática estruturada e com a parceria entre os envolvidos. Os integrantes da equipe devem trabalhar em consonância com os limites e especificidades de sua profissão, e respeitada estas particularidades, precisam conhecer a ação individual de cada integrante do grupo. Dessa forma, sua prática favorecerá ao paciente um panorama do problema, dando-lhes informação e estímulo para superar os obstáculos e adotar comportamentos que modifiquem os hábitos de vida e cooperem com a real adesão à terapêutica proposta, de forma continuada e individualizada para cada paciente (MALACHIAS et al., 2017).

Abordagem biopsicossocial: As manifestações da doença podem influenciar tanto no bem-estar como nos seus vínculos familiares e sociais (MOREIRA et al., 2010). Sintomas que influenciam de modo direto na imagem, podem provocar uma redução no prazer sexual e também interferir na identidade feminina, devido ao sentimento de estigmatização, por grande parte delas não corresponderem aos padrões de beleza impostos pela sociedade (SNYDER, 2006 apud MOREIRA et al., 2013; CHING; BURKE; STUCKEY, 2007 apud MOREIRA et al., 2013). A obesidade, por exemplo, vem sendo considerada como um dos sintomas que mais implica na redução da qualidade de vida dessas mulheres, estando ligada a sensação de tristeza e ansiedade (TANG et al., 2006; MOREIRA et al., 2013). A acne e o hirsutismo estão correlacionados como as causas mais prevalentes de estresse emocional e social (JONES et al., 2008), enquanto a infertilidade e os distúrbios menstruais precedem o estresse psicológico e a redução do bem-estar (LI et al., 2011) possivelmente pela sensação de estigmatização (MOREIRA et al., 2010) e pela grande angústia que a infertilidade carrega consigo, ampliando assim a prevalência do transtorno depressivo nessa população (DEEKS, 2010). Demonstra-se também a imprescindibilidade da inserção de dermatologistas e outros profissionais da área da estética para o tratamento do hirsutismo, acne, seborreia, alopecia e demais manifestações da SOP que impactam negativamente na aparência dessas mulheres. Essas terapêuticas podem ser aplicadas de forma individual ou em associação ao tratamento medicamentoso em quadros de gravidade moderada ou grave (MOREIRA et.al., 2010; SILVA-DE-SÁ, 2018). Mesmo com a enorme repercussão que os sintomas da SOP acarretam na vida de suas portadoras, repercutindo na vertente psicossocial, a maioria das pesquisas tem sido elaboradas em uma concepção biológica, não sendo apropriado para analisar a complexidade da vivência dessas mulheres, o que comprova a necessidade de estudos psicossociais (MOREIRA et al., 2013; SNYDER, 2006 apud MOREIRA et al., 2013). Vale ressaltar que a valorização e reconhecimento dos sentimentos derivados dos seus sintomas podem auxiliar de forma significativa na adesão ao tratamento indicado. A simples notificação da necessidade de haver uma mudança no estilo de vida não tem se mostrado muito eficaz, é preciso também criar um plano terapêutico conforme a realidade e as possibilidades da paciente, levando em conta os sentimentos de ansiedade existentes (MOREIRA et al., 2013). Dessa forma, acredita-se que grupos terapêuticos específicos para pacientes com SOP podem ser benéficos por ajudar a criar métodos psicológicos saudáveis para $o$ enfrentamento da sua condição e realidade. Assim, elas terão mais motivação para modificar seus hábitos, praticando assim exercícios físicos regulares e participando de programas de reeducação alimentar, podendo assim alcançar de fato uma vida de qualidade (MOREIRA et al., 2013). Dessa forma, torna-se imperativo que seja ofertado tratamento psicológico à essas pacientes, não deixando que as demandas psicoemocionais sejam desprezadas, auxiliando-as assim a obter a atenção de profissionais capacitados, os quais podem a ajudar a recuperar sua autoestima e proporcionar uma qualidade de vida satisfatória. Para isso, as melhores abordagens psicológicas para esse tratamento específico são a Terapia Cognitivo-Comportamental e a Análise Comportamental, pois possuem evidências empíricas de suas eficácias, além de proporcionar mudanças no emocional e na conduta de forma rápida, se equiparado com as outras linhas deste campo do saber (NEUENFELDT, 2014).

Exercício físico: A SOP configura um modelo biológico multifacetado no que se relaciona com o perfil hormonal e o risco cardiovascular. Nessas mulheres, a associação entre RI e um fenótipo de sedentarismo origina um perfil de risco cardiovascular ainda mais prejudicial, com propensão à elevação do IMC, baixa capacidade cardiopulmonar, diminuição da aptidão física e estado pró-inflamatório crônico elevado (VIGORITO et al., 2007). Nesse contexto, uma das melhores táticas do tratamento para a obesidade, hiperandrogenismo e infertilidade para as mulheres com SOP é a pratica frequente de exercício físico (HUBER-BUCHHOLZ; CAREY; NORMAN, 1999; VIGORITO et al., 2007). A qual representa um modulador benéfico dos determinantes de risco cardiovascular nessas pacientes (RANDEVA et al., 2002), sendo indispensável a sua realização (AZEVEDO et al., 2008). Mesmo com toda sua importância clínica, sua pratica tem sido pouco utilizada como recurso terapêutico, encontrando-se um quantidade reduzida de trabalhos científicos com esse intuito. Assim como, não há uma investigação acerca do tipo, intensidade, frequência, duração e progressão dessa prática nessas mulheres (AZEVEDO et al., 2008).

Efeitos sobre a composição corporal: Pacientes com SOP que apresentam sobrepeso possuem um alto índice de obesidade central, fator que se associa de modo direto com a RI aumentada (CASCELLA et al., 2008). Estudo realizado por Huber-Huber-Buchholz, Carey e Norman (1998), demonstrou que, portadoras obesas da síndrome após realizar seis meses de exercício e dieta, alcançaram uma modificação favorável na composição corporal, com diminuição da circunferência da cintura, da insulina basal e do nível de LH, além de uma melhora na sensibilidade à insulina, mesmo com baixo nível de perda da massa corporal total (2 a 5\%). No entanto, tais benefícios só foram vistos nas pacientes responsivas à 
intervenção, ou seja, as que se tornaram ovulatórias no decorrer e/ou no final da pesquisa. Ter uma redistribuição da massa corporal gorda tem uma importância maior do que sua perda, possuindo impacto positivo na restauração da função ovariana e na sensibilidade à insulina (HUBER-HUBERBUCHHOLZ, CAREY, NORMAN, 1998). Em estudo feito por HOEGER et al. (2004), foi constatado que a combinação entre dieta, exercício e metformina em mulheres com SOP, resulta em uma redução da massa corporal total mais expressiva quando comparada com as abordagens isoladas. Os resultados das pesquisas existentes recomendam o incentivo a prática regular e continua de exercício físico, visto que a interrupção mesmo que por um período de tempo curto pode acarretar na supressão dos benefícios alcançados previamente (AZEVEDO et al., 2008).

Parâmetros cardiovasculares: Um dos fatores de risco cardiovascular notado foram os níveis elevados de homocisteína plasmática em mulheres jovens com sobrepeso e obesas com SOP. Estudo realizado por Randeva et al. (2002), verificou que as mulheres que adotaram o programa de exercício proposto tiveram uma redução na concentração de homocisteína plasmática, além de uma melhora no consumo máximo de oxigênio (VO2 max). Esses desfechos são importantes, pois ressaltam como o exercício físico pode influenciar sobre esse marcador clínico extremamente pertinente (VO2 max), o qual se relaciona de forma negativa com a mortalidade cardiovascular e, de forma não direta, com todas as demais causas (BLAIR et al., 1995; ERIKSSEN et al., 1998; LAMONTE et al., 2000; LAUKKANEN et al., 2001; KAVANAGH et al., 2002). A elevação do VO2 max visto posteriormente à prática aeróbica nessas mulheres aparenta se associar com a diminuição do IMC, circunferência da cintura, relação cintura-quadril e área sob a curva de insulina, além de aumento na relação glicose-insulina (VIGORITO et al., 2007). No mesmo viés de pesquisa, notou-se uma evolução na capacidade máxima de trabalho, elevação do consumo de oxigênio no limiar anaeróbico, redução da frequência cardíaca de repouso, da pressão arterial sistólica de repouso, da pressão arterial diastólica máxima e do nível basal de proteína $\mathrm{C}$ reativa após três meses de exercício aeróbico continuo (VIGORITO et al., 2007). Outras descobertas foram o aumento no nível de atividade física e no gasto calórico diário dessas mulheres, incluindo atividades recreacionais e domésticas, tendo esses aspectos se relacionando positivamente, com melhora no VO2 max, área sob a curva de insulina e relação glicose-insulina.

Parâmetros bioquímicos e hormonais: Hoeger et al. (2004) evidenciaram que a associação de metformina, dieta e prática regular de exercício conseguiu diminuir 4,5\% nos níveis de testosterona plasmática e de 15,2\% nos de androgênios livres (FAI). Outra pesquisa também demonstrou a elevação do SHBG e, a diminuição dos índices de sulfato de dehidroepiandrosterona (DHEA-S) e androstenediona plasmática (PALOMBA et al., 2008). Por ajudar a utilização periférica da glicose e aprimorar a sensibilidade à insulina, a prática regular de exercícios físicos está relacionada à redução nos níveis de insulina de jejum nas portadoras da SOP (BRUNER; CHAD; CHIZEN, 2006). No que se diz respeito ao perfil lipídico, a prática continua de exercícios físicos consegue reduzir de forma expressiva os níveis da lipoproteína de baixa densidade (LDL) e elevar a lipoproteína de alta densidade (HDL), relacionando esse fato com o tempo de treinamento (ORIO et al., 2008).
Necessita-se de mais investigações para elucidar as repercussões dos exercícios físicos sobre os indicadores clínicos e hormonais do hiperandrogenismo (AZEVEDO et al., 2008).

Função reprodutiva: Uma das metas mais relevantes na terapêutica da SOP é a restauração da função ovulatória (AZEVEDO et al., 2008). Em pesquisa realizada por Hoeger et al. (2004), foi notada uma propensão a maior periodicidade de ovulações regulares, a qual foi demonstrada pela excreção urinária de pregnanediol, nos grupos experimentais que combinavam dieta, exercício e metformina, quando comparado ao grupo controle. $\mathrm{O}$ grupo que perdeu $3 \%$ ou mais de massa corporal manifestou nove vezes mais chance de ovular regularmente. Vinculando essa conduta ao uso de metformina, esse índice sobe para 16 vezes. Tais indicadores correlacionados com as considerações de outras pesquisas (VIGORITO et al., 2007) são importantes para comprovar o impacto positivo que tem o exercício físico (seja isolado ou em associação com dieta e/ou metformina) sobre a melhora da função reprodutiva dessas pacientes.

Medicamentos: Ainda não se sabe com exatidão se pode ser utilizada a medicação como tratamento preventivo de sintomas iniciais ou como cura da patologia. Desse modo, a recomendação de seu uso deve ter como objetivo a melhora do quadro visando evitar complicações (THE ROTTERDAM ESHRE/ASRM-SPONSORED PCOS CONSENSUS WORKSHOP GROUP, 2004). Entre as terapêuticas medicamentosas recomendadas para essas pacientes, podemos ressaltar a metformina, medicação antidiabética oral, o anticoncepcional, medicação contraceptiva composta, na maioria das vezes, por dois hormônios (estrogênio e progestina), o mio-inositol $(\mathrm{MI})$, suplemento sensibilizante à insulina, os antiandrogênicos e os indutores de ovulação (CONSTANTINO, MINOZZI e GUARALDI, 2009; SPRITZER et al., 2014).

Anticoncepcionais orais combinados (AOCs): Os AOCs são estro-progestinas largamente utilizadas como possibilidade terapêutica em mulheres com hirsutismo moderado ou grave ou com outra sintomatologia hiperandrogênica, que não possuem o desejo engravidar. Eles atuam suprimindo a liberação de LH e aumentando a produção do SHBG, reduzindo consequentemente a concentração de androgênios livres no plasma (ROCCA et al., 2015 apud SILVA et al., 2020; ARENTZ et al., 2017; SILVA et al., 2020). Os AOCs mais utilizados nos dias de hoje são os de baixa dose, contendo etinilestradiol e progestinas antiandrogênicas (ROCCA et al., 2015 apud SILVA et al., 2020). Quando os AOCs são contraindicados, outras opções à disposição são as pílulas anticoncepcionais contendo apenas progestinas ou dispositivos intrauterinos (SPRITZER et al., 2014). Mesmo sendo os medicamentos de primeira linha para tratar essas mulheres, eles possuem efeitos metabólicos prejudiciais. Dentre eles, verifica-se que a terapia hormonal contendo progestinas androgênicas eleva o risco do desenvolvimento de eventos cardiovasculares e tromboembólicos (MOURA, 2011). Ademais, pesquisas demonstram que eles ocasionam a elevação do colesterol total e do LDL, redução do HDL e podem aumentar os triglicerídeos, predispondo ou agravando o quadro de dislipidemia (FERREIRA; D'AVILA; SAFATLE, 2019). Existem comprovações que demonstram que a associação do uso dos AOCs com as mudanças no estilo de vida, propiciando a perda de peso, pode potencialmente 
suprimir os efeitos metabólicos adversos desses medicamentos (LEGRO RS, et al., 2015). Portanto, é essencial ponderar os riscos e benefícios para cada paciente quando for utilizar a terapia hormonal (SILVA et al., 2020).

Antiandrogênios: Os antiandrogênicos são uma classe de fármacos encarada como modelo para tratar $\mathrm{O}$ hiperandrogenismo, apresentado na SOP, podendo ser utilizada em conjunto com os AOCs ou usadas como opção após a falha no tratamento do uso de AOCs por pelo menos 6 meses (ROCCA et al., 2015 apud SILVA et al., 2020; SPRITZER et al., 2014). No emprego da terapia androgênica deve ser utilizado de forma obrigatória a contracepção em razão das suas implicações teratogênicas (SPRITZER et al., 2014). Os antiandrogênios mais relevantes à disposição de forma isolada são o acetato de ciproterona, espironolactona, finasterida e flutamida (FEBRASGO, 2018). A espironolactona é o fármaco mais usado entre elas, sendo um antagonista seguro e eficiente na atenuação do hirsutismo (JIN; XIE, 2018 apud SILVA et al., 2020). A utilização dela inibe as complicações cardiovasculares, em razão da redução dos andrógenos e a sua influência no sistema renina-angiotensina-aldosterona que está envolvido no mecanismo de formação de placas aterogênicas que pode ser provocada pela SOP (ROCCA et al., 2015 apud SILVA et al., 2020). Todas essas terapêuticas sistêmicas devem ser realizadas de forma prolongada, fato que deve ser advertido para a paciente, não se devendo reavaliar esses casos antes de 6 meses de tratamento, em particular para a queixa de hirsutismo, em razão deles funcionarem encurtando a fase anágena do folículo. Uma outra questão para se ressaltar é que os desfechos são superiores e talvez perceptíveis em prazos menores quando se associa tratamentos, ou seja, a combinação de diferentes drogas e/ou tratamentos cosméticos. Sendo que nas pacientes obesas, a eficácia do tratamento pode ser mais limitada (FEBRASGO, 2018).

Drogas sensibilizadoras da insulin: Pesquisas comprovaram que a RI é restrita às mulheres com anovulação crônica e hiperandrogenismo e é independente da obesidade, sendo ligada a uma redução de $35 \%$ na ação da insulina, e, nas pacientes obesas, ocorre um decréscimo de $50 \%$ em sua ação (SILVEIRA, 2008). Nesse contexto, a utilização de sensibilizadores da insulina passou a ser recomendada no tratamento da SOP após a confirmação de que a RI possui um papel importante em sua fisiopatologia (SIVALINGAM, 2014).

Metformina: A metformina e as tiazolidinedionas são os fármacos sensibilizadores da insulina mais usados em mulheres com SOP, sendo eficientes na diminuição do hiperandrogenismo. Entretanto estas não são muito efetivas no hirsutismo, um dos sintomas desta patologia. A metformina é a mais utilizada devido aos efeitos colaterais que as tiazolidinedionas (pioglitazona e rosiglitazona) carregam consigo como o ganho de peso e risco para câncer (SPRITZER et al., 2014). Entre os efeitos benéficos que a metformina possui podemos citar à redução nos níveis de androgênios, agindo na atenuação do risco cardiovascular, na melhora do índice de RI, o que evita a tolerância à glicose, possuindo fatores positivos no diabetes, e, faz com que reduzam os índices de progressão para a doença em mulheres prédiabéticas (SPRITZER et al., 2014). Ademais, essa droga possui um efeito positivo sobre a pressão arterial, ocasionando melhora na função vascular, isso ajuda na prevenção e no controle da dislipidemia, condição comum as pacientes, visto que atua na redução dos níveis de triglicerídeos, colesterol LDL e aumenta o HDL (ORTIZ-FLORES et al., 2018 apud SILVA et al., 2020; ROCCA et al., 2015 apud SILVA et al., 2020). Sua ação no que diz respeito a fertilidade é questionável, não aparentando ser tão funcional em pacientes obesas. Nesse sentido, a metformina não é mais vista como um tratamento de primeira linha para a fertilidade na grande maioria dos casos (JOHNSON et al., 2010; SPRITZER et al., 2015).

Mio-inositol (MI): O Mio-inositol (MI) é o isômero de inositol mais numeroso dentro do ovário, e sua carência local pode ser o causador da oligoovulação e da baixa qualidade de ovócitos no distúrbio. Isso está ligado de forma direta com o fato dele gerar maturação nas células germinativas femininas (CHIU et al., 2002). Essa desregulação no metabolismo do inositol, se conecta também com a RI. Sua suplementação demonstra possuir efeitos na frequência da ovulação, na redução da concentração de hormônios androgênios e na concentração sérica insulina, e na prevenção de patologias associadas à síndrome (NORDIO e PROIETTI, 2012; UNFER et al., 2016; COMISSÃO NACIONAL DE INCORPORAÇÃO DE TECNOLOGIAS NO SUS, 2019). A dose indicada varia de $200 \mathrm{mg}$ a $4 \mathrm{~g}$ de $\mathrm{MI}$ por dia nas pesquisas analisadas (UNFER et al., 2016). O tempo de uso é essencial para sua eficácia, sendo estimado em pelo menos 12 semanas para atingir os resultados almejados (JONAS, 2019).

Indução da ovulação: A primeira linha de terapêutica medicamentosa para a infertilidade anovulatórias nas pacientes com SOP, com desejo reprodutivo, é a indução da ovulação. Dentre os fármacos à disposição, o citrato de clomifeno (CC) é considerado o melhor, por possuir eficácia em restaurar a ovulação (MORGANTE et al., 2017 apud SILVA et al., 2020; SPRITZER et al., 2014). Com o uso da dose terapêutica ideal de CC, em média 150 mg, a ovulação acontece em 60-85\% dos casos, com uma taxa de gravidez de 30 a 50\% após seis ciclos de ovulações (ROCCA et al., 2015 apud SILVA et al., 2020). Nos casos que o CC não possui êxito, as gonadotrofinas exógenas e a fertilização in vitro são as próximas opções viáveis para a infertilidade, seguida da diatermia ovariana laparoscópica, a qual estimula uma melhora dos ciclos menstruais e da ovulação em 50\% das pacientes (SILVA et al., 2020).

Abordagem nutricional: A principal abordagem no tratamento nas mulheres diagnosticadas com SOP que possuem sobrepeso ou são obesas é a reeducação alimentar, com o objetivo de perca de peso, com dieta hipocalórica, redução de gordura saturada e aumento de gordura mono e poli-insaturada. Assim como, aumento do consumo de fibras, frutas, cereais e vegetais (PONTES; ALMEIDA FILHO, 2016). O valor nutricional da dieta pode influenciar nas alterações endócrinas e metabólicas vigentes nessas pacientes, apesar de que tenha sido realizadas poucas pesquisas sobre esse assunto (PASQUALI; CASIMIRRI, 1993). Há, de fato, uma associação complexa de inter-relação entre diferentes fatores nutricionais e condições endócrinas. A dieta exerce um papel essencial na regulação do metabolismo dos esteroides sexuais e na secreção do LH (GAMBINERI et al., 2002). A redução de carboidratos foi relacionada com melhora de fatores de riscos coronarianos (FOSTER et al., 2003). Com isso, vem se gerando um interesse crescente por dietas para perca de peso que se propõe a modificar o perfil de macronutrientes no tratamento dessas pacientes, tais como 
dietas hiperprotéicas com diminuição, modificação ou restrição de carboidratos. Estas dietas colaboram para a redução mais eficaz do peso, ligada ao maior poder de saciedade das proteínas, se confrontada com os carboidratos e lipídios (MIKKELSEN; TOUBRO; ASTRUP, 2000). A alta ingestão de lipídios aparenta reduzir os níveis da SHBG, elevando, consequentemente, a disponibilidade de androgênios e estrogênios no tecido alvo (GAMBINERI et al., 2002; SANTOS et al., 2019). Acredita-se que uma alimentação com baixo teor de fibras possa levar ao aumento dos níveis de estrogênio e androgênios circulantes, por esse motivo é indicado uma dieta rica em fibras afim de reverter esse quadro (GAMBINERI et al., 2002; SANTOS et al., 2019). Além disso, o consumo de fibra foi relacionado de forma significativa com o aumento do nível do SHBG e com a normalização da concentração da insulina circulante (WILD et al., 2000 apud SANTOS et al., 2019). Ademais, várias pesquisas demonstram que quando a sensibilidade à insulina é elevada o peso corporal é diminuído em indivíduos com dietas ricas em fibras (FUKAGAWA et al., 1990 apud SANTOS et al., 2019).

Suplementação Nutricional: Além da análise de diferentes quantidades de macronutrientes da dieta, algumas pesquisas já relataram os benefícios da suplementação nas mulheres com SOP como a vitamina D e do cálcio na função hormonal (PAL et al., 2012; TEHRANI; MOSTAJERAN; SHAHSAVARI, 2014), do ômega-3 na melhoria da função da sensibilidade à insulina (RAFRAF et al., 2012), dos ácidos graxos poliinsaturados (PUFA) na modulação do perfil androgênicos (PHELAN et al., 2011), da Coenzima Q10, da vitamina E, do magnésio, do zinco, do picolinato de cromo, da melatonina, dentre outros.

Vitamina D e Ácido graxo ômega-3: As portadoras de SOP possuem um alta prevalência de deficiência de vitamina $D$, esse fato se correlaciona com vários sintomas da SOP, incluindo RI, infertilidade e hirsutismo, indicando que a vitamina $\mathrm{D}$ pode possuir um papel na patogênese da síndrome (THOMSON; SPEDDING; BUCKLEY, 2012; FANG et al., 2017). Em estudo realizado por Kotsa, Yavropoulou e Anastasiou (2009), notou-se uma melhoria na função reprodutiva dessas pacientes (elevação das taxas de gravidez, melhora da frequência menstrual, regulação dos ciclos menstruais), redução da testosterona livre, elevação da SHBG, ou seja, houve melhora do hiperandrogenismo, como, também, foi notado que, com a suplementação de $25(\mathrm{OH}) \mathrm{D}$, aumentou a secreção de insulina, consequentemente, melhora da RI nessas mulheres, além de haver aumento das concentrações de HDL e diminuição dos triglicerídeos, reduzindo, assim, os riscos de estas mulheres desenvolverem doenças cardiovasculares (RAZAVI et al., 2016). Em pesquisa realizada por Jamilian et al. (2018), notou-se que ao fazer uma co-suplementação de Vitamina D com o ácido graxo ômega-3 por 12 semanas obteve-se vários efeitos benéficos como a redução significativa dos níveis séricos de testosterona total, da PCR de alta sensibilidade sérica, de malondialdeído, da expressão gênica de interleucina-1 (IL-1) e do fator de crescimento endotelial vascular regulado positivamente. Além disso obteve-se uma melhora substancial nos níveis de capacidade antioxidante total plasmática e nos paramentos de saúde mental, com melhora nas pontuações do questionário de saúde geral e nos escores da escala de depressão, ansiedade e estresse.
Ácidos graxos poli-insaturados: A ingestão de alimentos com alto teor de ácidos graxos poli-insaturados (PUFAs) costuma ser indicado para mulheres com SOP. Evidências experimentais indicam que os PUFAs melhoram a ação da insulina em tecidos periféricos e reduzem a secreção de insulina pelo pâncreas (KATSIKIS et al., 2011 apud SANTOS et al., 2019).

Coenzima Q10 e / ou vitamina E: Em estudo realizado por Izadi et al. (2019), notou-se que ao combinar a suplementação da Coenzima Q10 (CoQ10) com a Vitamina E obteve-se uma redução substancial nos triglicerídeos séricos, nos níveis de colesterol total do soro, no LDL, no coeficiente aterogênico e nos valores do índice de adiposidade visceral. Além disso, aumentou os níveis de HDL.

Co-suplementação de magnésio e zinco: Em ensaio clínico controlado randomizado realizado por Ebrahimi et al. (2018), verificou-se que a com a a co-suplementação de magnésio e zinco por 12 semanas foi obtido uma redução significativa da PCR sérica de alta sensibilidade, do carbonil de proteína (PCO), da IL-1 e do fator de necrose tumoral alfa (TNF- $\alpha$ ). Além disso, os níveis de capacidade antioxidante total plasmática foram significativamente elevados.

Picolinato de Cromo: Em uma revisão sistemática e metaanálise realizada por Fazelian et al. (2017), notou-se que a suplementação com picolinato de cromo resultou em efeitos benéficos a diminuição do IMC, insulina em jejum e testosterona livre em mulheres com SOP. Porém, não possuiu efeitos na redução da testosterona total, pontuação de Ferriman-Galwey, DHEA, FSH e LH.

Melatonina: Em um ensaio randomizado, duplo-cego e controlado por placebo realizado por Shabani et al. (2019), percebeu-se que a suplementação de melatonina reduziu expressivamente o Índice de Qualidade do Sono de Pittsburgh e o índice de Inventário de Depressão de Beck, possuindo assim efeitos benéficos nos parâmetros de saúde mental. Além disso, diminuiu significativamente também os níveis de insulina, do modelo de homeostase de avaliação da resistência à insulina (HOMA-IR), do índice de verificação de sensibilidade à insulina quantitativa (QUICKI), o colesterol total, LDL e a expressão gênica do receptor gama ativado por proliferador de peroxissoma (PPAR- $\gamma$ ) e o receptor de lipoproteína de baixa densidade (LDLR) entre as pacientes com SOP.

Cirurgia bariátrica: As mulheres obesas com SOP são comprovadamente beneficiadas pela realização de cirurgia bariátrica, devendo primeiro realizar uma mudança no estilo de vida e após a falha desta, a cirurgia torna-se uma opção. Uma metanálise recente apontou uma resolução da SOP em 96\% das pacientes que tiveram uma perda de peso induzida pela cirurgia bariátrica (ORTIZ-FLORES et al., 2018 apud SILVA et al., 2020). Pesquisas indicam uma melhora substancial na hiperandrogenemia e na disfunção ovulatória, afetando positivamente a fertilidade da paciente após a cirurgia bariátrica (JIN; XIE, 2018 apud SILVA et al., 2020; ORTIZFLORES et al., 2018 apud SILVA et al., 2020; SPRITZER et al., 2014). Dessa forma, a cirurgia torna-se uma opção bastante viável nessas pacientes. 


\section{CONCLUSÃO}

Diante da complexidade da SOP e dos seus amplos efeitos sobre a saúde da mulher, demonstra-se necessário um olhar integral sobre essa síndrome e o diagnóstico precoce. Como descrito, o tratamento perpassa os fármacos como os anticoncepcionais, os sensibilizadores à insulina, os antiandrogênicos e os indutores de ovulação e abrange, principalmente, os cuidados não medicamentosos. A mudança no estilo de vida é imperativa no acompanhamento da mulher portadora dessa síndrome. Nesse contexto, a equipe multiprofissional torna-se essencial. $O$ conjunto de profissionais composto por ginecologista, endocrinologista, dermatologista, psicólogo, nutricionista, educador físico possui como objetivo perda de peso, melhora da resposta à insulina, atividade física, mudança da composição corporal, autoestima e bem-estar mental (CONSTANTINO, MINOZZI e GUARALDI, 2009; SPRITZER et al., 2014; SILVA-DE-SÁ, 2018; SPEELMAN, 2019). Por fim, apesar da evolução nos conhecimentos sobre a SOP, ainda existem lacunas científicas na fisiopatologia, diagnóstico, acompanhamento e tratamento dessa síndrome. No que diz respeito ao tratamento multiprofissional, são necessário maiores esforços para evidenciar a importância dessa assistência integral.

\section{REFERÊNCIAS}

Arentz, Susan et al. 2017. Combined lifestyle and herbal medicine in overweight women with polycystic ovary syndrome (PCOS): A randomized controlled trial. Phytotherapy Research, v. 31, n. 9, p. 1330-1340.

Azevedo, George Dantas de et al. 2008. Modificações do estilo de vida na síndrome dos ovários policísticos: papel do exercício físico e importância da abordagem multidisciplinar. Rev. Bras. Ginecol. Obstet., Rio de Janeiro, v. 30, n. 5, p. 261-267, May 2008.

Benetti-Pinto, Cristina Laguna. Tratamento das manifestações androgênicas. Síndrome dos ovários policísticos. São Paulo: Federação Brasileira das Associações de Ginecologia e Obstetrícia (FEBRASGO), p. 56-67, 2018.

Blair, Steven N. et al. Changes in physical fitness and allcause mortality: a prospective study of healthy and unhealthy men. Jama, v. 273, n. 14, p. 1093-1098, 1995.

BRUNER, Brenda; CHAD, Karen; CHIZEN, Donna. Effects of exercise and nutritional counseling in women with polycystic ovary syndrome. Applied physiology, nutrition, and metabolism, v. 31, n. 4, p. 384-391, 2006.

Cascella, Teresa et al. Visceral fat is associated with cardiovascular risk in women with polycystic ovary syndrome. Human Reproduction, v. 23, n. 1, p. 153-159, 2008.

Ching, H. L., Burke, Valerie; Stuckey, B. G. A. Quality of life and psychological morbidity in women with polycystic ovary syndrome: body mass index, age and the provision of patient information are significant modifiers. Clinical endocrinology, v. 66, n. 3, p. 373-379, 2007.

Chiu, Tony TY et al. Follicular fluid and serum concentrations of myo-inositol in patients undergoing IVF: relationship with oocyte quality. Human reproduction, v. 17, n. 6, p. 1591-1596, 2002.

Comissão Nacional DE Incorporação DE Tecnologias NO SUS. Protocolo Clínico e Diretrizes Terapêuticas para Síndrome dos Ovários Policísticos. Conitec, 2019. Disponível
http://conitec.gov.br/images/Consultas/2019/Relatorio_PC DT_SindromeOvariosPolicisticos_CP05_2019.pdf Acesso: 15 fev. 2020.

Costantino, D. et al. Metabolic and hormonal effects of myoinositol in women with polycystic ovary syndrome: a double-blind trial. Eur Rev Med Pharmacol Sci, v. 13, n. 2, p. 105-110, 2009.

Da Silveira, Gustavo Py Gomes. Ginecologia baseada em evidências. Atheneu, 2008.

Deeks, Amanda A., Gibson-Helm, Melanie E., Teede, Helena J. Anxiety and depression in polycystic ovary syndrome: a comprehensive investigation. Fertility and sterility, v. 93, n. 7, p. 2421-2423, 2010.

Ebrahimi, Faraneh Afshar et al. The effects of magnesium and zinc co-supplementation on biomarkers of inflammation and oxidative stress, and gene expression related to inflammation in polycystic ovary syndrome: a randomized controlled clinical trial. Biological trace element research, v. 184 , n. 2 , p. $300-307,2018$.

Erikssen, Gunnar et al. Changes in physical fitness and changes in mortality. The Lancet, v. 352, n. 9130, p. 759$762,1998$.

Fang, Fang et al. Effect of vitamin D supplementation on polycystic ovary syndrome: A systematic review and metaanalysis of randomized controlled trials. Complementary Therapies in Clinical Practice, v. 26, p. 53-60, 2017.

Fazelian, Siavash et al. Chromium supplementation and polycystic ovary syndrome: A systematic review and metaanalysis. Journal of Trace Elements in Medicine and Biology, v. 42, p. 92-96, 2017.

Ferreira, Laura Fernandes; D’AVILA, A. M. F. S., Safatle, Giselle Cunha Barbosa. O uso da pílula anticoncepcional e as alterações das principais vias metabólicas. Femina.[Internet], v. 47, n. 7, p. 426-32, 2019.

Foster, Gary D. et al. A randomized trial of a lowcarbohydrate diet for obesity. New England Journal of Medicine, v. 348, n. 21, p. 2082-2090, 2003.

Fukagawa, Naomi K. et al. High-carbohydrate, high-fiber diets increase peripheral insulin sensitivity in healthy young and old adults. The American journal of clinical nutrition, v. 52, n. 3, p. 524-528, 1990.

Gambineri, A. et al. Obesity and the polycystic ovary syndrome. International journal of obesity, v. 26, n. 7, p. 883-896, 2002.

Goodman, Neil F. et al. American Association of Clinical Endocrinologists, American College of Endocrinology, and androgen excess and PCOS society disease state clinical review: guide to the best practices in the evaluation and treatment of polycystic ovary syndrome-part 1. Endocrine Practice, v. 21, n. 11, p. 1291-1300, 2015.

Hoeger, Kathleen M. et al. A randomized, 48-week, placebocontrolled trial of intensive lifestyle modification and/or metformin therapy in overweight women with polycystic ovary syndrome: a pilot study. Fertility and sterility, v. 82, n. 2, p. 421-429, 2004.

Huber-Buchholz, M.-M., Carey, D. G. P., Norman, R. J. Restoration of reproductive potential by lifestyle modification in obese polycystic ovary syndrome: role of insulin sensitivity and luteinizing hormone. The Journal of Clinical Endocrinology \& Metabolism, v. 84, n. 4, p. 14701474, 1999.

Izadi, Azimeh et al. Independent and Additive Effects of Coenzyme Q10 and Vitamin E on Cardiometabolic Outcomes and Visceral Adiposity in Women With 
Polycystic Ovary Syndrome. Archives of medical research, v. 50, n. 2, p. 1-10, 2019.

Jamilian, Mehri et al. The influences of vitamin D and omega3 co-supplementation on clinical, metabolic and genetic parameters in women with polycystic ovary syndrome. Journal of affective disorders, v. 238, p. 32-38, 2018.

JIN, Peipei; XIE, Yongyong. Treatment strategies for women with polycystic ovary syndrome. Gynecological Endocrinology, v. 34, n. 4, p. 272-277, 2018.

Johnson, N. P. et al. PCOSMIC: a multi-centre randomized trial in women with PolyCystic Ovary Syndrome evaluating Metformin for Infertility with Clomiphene. Human reproduction, v. 25, n. 7, p. 1675-1683, 2010.

Jonas, Camila Feksa. Uso terapêutico de mio-inositol como tratamento em pacientes com síndrome do ovário policístico. 2019.

Jones, G. L. et al. Health-related quality of life measurement in women with polycystic ovary syndrome: a systematic review. Human reproduction update, v. 14, n. 1, p. 15-25, 2008.

Katsikis, Ilias et al. Phenotypic expression, body mass index and insulin resistance in relation to $\mathrm{LH}$ levels in women with polycystic ovary syndrome. European Journal of Obstetrics \& Gynecology and Reproductive Biology, v. 156, n. 2, p. 181-185, 2011.

Kavanagh, Terence et al. Prediction of long-term prognosis in 12169 men referred for cardiac rehabilitation. Circulation, v. 106, n. 6, p. 666-671, 2002.

Kotsa, K., Yavropoulou, M.P., Anastasiou, O. Role of vitamin $\mathrm{D}$ treatment in glucose metabolism in polycystic ovary syndrome. Fertility and sterility, v. 92, n. 3, p. 1053-1058, 2009.

Lamonte, Michael J. et al. Cardiorespiratory fitness and coronary heart disease risk factors: the LDS Hospital Fitness Institute cohort. Circulation, v. 102, n. 14, p. 1623$1628,2000$.

Laukkanen, Jari A. et al. Cardiovascular fitness as a predictor of mortality in men. Archives of internal medicine, v. 161, n. 6, p. 825-831, 2001.

Legro, Richard S. et al. Randomized controlled trial of preconception interventions in infertile women with polycystic ovary syndrome. The Journal of Clinical Endocrinology \& Metabolism, v. 100, n. 11, p. 4048-4058, 2015.

Li, Yanan et al. Polycystic ovary syndrome is associated with negatively variable impacts on domains of health-related quality of life: evidence from a meta-analysis. Fertility and sterility, v. 96, n. 2, p. 452-458, 2011.

Malachias, M.V. et al. $7^{\mathrm{a}}$ Diretriz Brasileira de Hipertensão Arterial. Revista Brasileira de Hipertensão, v.24, n.1, 2017. Disponível em: http://departamentos.cardiol.br/sbcdha/profissional/revista/24-1.pdf. Acesso em 01 jun. 2020.

Martins, Gilberto de Andrade; PINTO, Ricardo Lopes. Manual para elaboração de trabalhos acadêmicos. São Paulo: Atlas, 2001.

Mikkelsen, Per B., TOUBRO, Søren; ASTRUP, Arne. Effect of fat-reduced diets on 24-h energy expenditure: comparisons between animal protein, vegetable protein, and carbohydrate. The American journal of clinical nutrition, v. 72, n. 5, p. 1135-1141, 2000.

Moreira, Simone da Nóbrega Tomaz et al. Quality of life and psychosocial aspects of polycystic ovary syndrome: a quali-quantitative approach. Revista Brasileira de Ginecologia e Obstetrícia, v. 35, n. 11, p. 503-510, 2013.
Moreira, Simone et al. Síndrome de ovários policísticos: enfoque psicossocial. 2010.

Morgante, G. et al. Therapeutic approach for metabolic disorders and infertility in women with PCOS. Gynecological Endocrinology, v. 34, n. 1, p. 4-9, 2018.

Moura, Heloisa Helena Gonçalves de et al. Síndrome do ovário policístico: abordagem dermatológica. Anais Brasileiros de Dermatologia, v. 86, n. 1, p. 111-119, 2011.

Neuenfeldt, Gabriele Azevedo. A terapia cognitivocomportamental como possibilidade e desafio na gestão do atendimento psicológico no SUS. 2014.

Nordio, M., Proietti, E. The combined therapy with myoinositol and D-chiro-inositol reduces the risk of metabolic disease in PCOS overweight patients compared to myoinositol supplementation alone. Eur Rev Med Pharmacol Sci, v. 16, n. 5, p. 575-581, 2012.

Orio, Francesco et al. Metabolic and cardiopulmonary effects of detraining after a structured exercise training programme in young PCOS women. Clinical endocrinology, v. 68, n. 6, p. 976-981, 2008.

Ortiz-Flores, Andrés E., LUQUE-RAMÍREZ, Manuel; ESCOBAR-MORREALE, Héctor F. Pharmacotherapeutic management of comorbid polycystic ovary syndrome and diabetes. Expert Opinion on Pharmacotherapy, v. 19, n. 17, p. 1915-1926, 2018.

PAL, Lubna et al. Therapeutic implications of vitamin D and calcium in overweight women with polycystic ovary syndrome. Gynecological Endocrinology, v. 28, n. 12, p. 965-968, 2012.

PALOMBA, S. et al. Structured exercise training programme versus hypocaloric hyperproteic diet in obese polycystic ovary syndrome patients with anovulatory infertility: a 24week pilot study. Human reproduction, v. 23, n. 3, p. 642650, 2008.

PASQUALI, Renato; CASIMIRRI, Francesco. The impact of obesity on hyperandrogenism and polycystic ovary syndrome in premenopausal women. Clinical endocrinology, v. 39, n. 1, p. 1-16, 1993.

PEDUZZI, Marina. Equipe multiprofissional de saúde: conceito e tipologia. Revista de saúde pública, v. 35, n. 1, p. 103-109, 2001.

PHELAN, Niamh et al. Hormonal and metabolic effects of polyunsaturated fatty acids in young women with polycystic ovary syndrome: results from a cross-sectional analysis and a randomized, placebo-controlled, crossover trial-. The American journal of clinical nutrition, v. 93, n. 3, p. 652-662, 2011.

PONTES, A. G., ALMEIDA FILHO, B. S. Síndrome dos Ovários Policísticos: diagnóstico, tratamento e repercussões ao longo da vida. In: SÍNDROME dos Ovários Policísticos: diagnóstico, tratamento e repercussões ao longo da vida. Botucatu: Unesp, 2016. p. $1-32$.

RAFRAF, Maryam et al. Omega-3 fatty acids improve glucose metabolism without effects on obesity values and serum visfatin levels in women with polycystic ovary syndrome. Journal of the American College of Nutrition, v. 31, n. 5, p. 361-368, 2012.

RANDEVA, Harpal S. et al. Exercise decreases plasma total homocysteine in overweight young women with polycystic ovary syndrome. The Journal of Clinical Endocrinology \& Metabolism, v. 87, n. 10, p. 4496-4501, 2002.

RAZAVI, M. et al. The effects of vitamin DK-calcium cosupplementation on endocrine, inflammation, and oxidative stress biomarkers in vitamin D-deficient women with 
polycystic ovary syndrome: a randomized, double-blind, placebo-controlled trial. Hormone and Metabolic Research, v. 48, n. 07, p. 446-451, 2016.

ROCCA, Morena Luigia et al. Polycystic ovary syndrome: chemical pharmacotherapy. Expert opinion on pharmacotherapy, v. 16, n. 9, p. 1369-1393, 2015.

ROSA-E-SILVA AC. Conceito, epidemiologia e fisiopatologia aplicada à prática clínica. Síndrome dos ovários policísticos. São Paulo: Federação Brasileira das Associações de Ginecologia e Obstetrícia (FEBRASGO), p. 1-15, 2018.

SANTOS, Thaís Silva et al. ASPECTOS NUTRICIONAIS E MANEJO ALIMENTAR EM MULHERES COM SÍNDROME DOS OVÁRIOS POLICÍSTICOS. Revista Saúde em Foco, [S. L.], v. 11, p. 649-670, 2019. Disponível em: https://p ortal.unisepe.com.br/unifia/wpcontent/uploads/sites/1000 1/2019/06/058_ASPECTOSNUTRICIONAIS-E-MA NEJO-ALIMENTAR-EMMULHERES-COM-S \%C3\%8DNDROME-DOSOV\%C3\%81RIOS-POLIC\%C3\%8D STICOS 64 9_a_670.pdf. Acesso em: 30 set. 2020.

SHABANI, Azade et al. Effects of melatonin administration on mental health parameters, metabolic and genetic profiles in women with polycystic ovary syndrome: a randomized, double-blind, placebo-controlled trial. Journal of Affective Disorders, v. 250, p. 51-56, 2019.

SILVA, Isabelle Salomão Teixeira et al. A associação da mudança no estilo de vida com a terapia farmacológica no tratamento da Síndrome dos Ovários Policísticos (SOP). Revista Eletrônica Acervo Saúde, n. 57, p. e3909-e3909, 2020.

SILVA-DE-SÁ, Marcos Felipe. Qualidade de vida em mulheres com SOP. Síndrome dos ovários policísticos. São Paulo: Federação Brasileira das Associações de Ginecologia e Obstetrícia (FEBRASGO), p. 40-55, 2018.

SIVALINGAM, Vanitha N. et al. Metformin in reproductive health, pregnancy and gynaecological cancer: established and emerging indications. Human reproduction update, v. 20, n. 6, p. 853-868, 2014.

SNYDER, Barbara S. The lived experience of women diagnosed with polycystic ovary syndrome. Journal of obstetric, gynecologic \& neonatal nursing, v. 35, n. 3, p. 385-392, 2006.

SPEELMAN, Diana L. Nonpharmacologic Management of Symptoms in Females With Polycystic Ovary Syndrome: A Narrative Review. J Am Osteopath Assoc, v. 119, n. 1, p. 25-39, 2019.
SPRITZER, Poli Mara. Polycystic ovary syndrome: reviewing diagnosis and management of metabolic disturbances. Arquivos Brasileiros de Endocrinologia \& Metabologia, v. 58, n. 2, p. 182-187, 2014.

TANG, Thomas et al. Combined lifestyle modification and metformin in obese patients with polycystic ovary syndrome. A randomized, placebo-controlled, double-blind multicentre study. Human reproduction, v. 21, n. 1, p. 8089, 2006.

TEHRANI, Hatav Ghasemi; MOSTAJERAN, Fatemeh; SHAHSAVARI, Somayeh. The effect of calcium and vitamin D supplementation on menstrual cycle, body mass index and hyperandrogenism state of women with poly cystic ovarian syndrome. Journal of research in medical sciences: the official journal of Isfahan University of Medical Sciences, v. 19, n. 9, p. 875, 2014.

THE ROTTERDAM ESHRE/ASRM-SPONSORED PCOS CONSENSUS WORKSHOP GROUP. Revised 2003 consensus on diagnosis criteria and long-term health risks related to polycystic ovary syndrome. Fertil Steril, v. 81, n. 1, p. 19-25, 2004.

THOMSON, Rebecca L., SPEDDING, Simon; BUCKLEY, Jonathan D. Vitamin D in the aetiology and management of polycystic ovary syndrome. Clinical endocrinology, v. 77, n. 3, p. 343-350, 2012.

TRENTINI, Mercedes; PAIM, Lygia. 1999. Pesquisa em enfermagem: uma modalidade convergente-assistencial. Florianópolis: UFSC, v. 162.

UNFER, Vittorio et al. 2016. Effects of inositol (s) in women with PCOS: a systematic review of randomized controlled trials. International journal of endocrinology, v. 2016.

VIGORITO, Carlo et al. 2007. Beneficial effects of a threemonth structured exercise training program on cardiopulmonary functional capacity in young women with polycystic ovary syndrome. The Journal of Clinical Endocrinology \& Metabolism, v. 92, n. 4, p. 1379-1384.

VILAR, Lucio et. al. Endocrinologia Clínica. 6 ed. Rio de Janeiro: Guanabara Koogan, 2016.

WILD, Sarah et al. 2000. Cardiovascular disease in women with polycystic ovary syndrome at long $\square$ term follow $\square$ up: a retrospective cohort study. Clinical endocrinology, v. 52, n. 5, p. 595-600. 